\title{
Robust Signatures of the Relic Neutrinos in CMB*
}

\author{
Sergei Bashinsky \\ International Centre for Theoretical Physics \\ Strada Costiera 11, 31014 Trieste, Italy \\ E-mail: bashinsky@ictp.trieste.it
}

\begin{abstract}
When the perturbations forming the acoustic peaks of the cosmic microwave background $(\mathrm{CMB})$ reentered the horizon and interacted gravitationally with all the matter, neutrinos presumably comprised $41 \%$ of the universe energy. CMB experiments have reached a capacity to probe this background of relic neutrinos. I review the neutrino impact on the $\mathrm{CMB}$ at the onset of the acoustic oscillations. The discussion addresses the underlying physics, robustness or degeneracy of the neutrino imprints with changes of free cosmological parameters, and non-minimal models for the unseen radiation sector with detectable signatures in CMB anisotropy and polarization.
\end{abstract}

\section{Introduction}

The counts of solar, atmospheric, reactor, and accelerator-produced neutrinos provide a solid evidence of physics beyond the Standard Model of particle physics. They can be explained by neutrino flavor mixing by non-zero masses, subject to tightening upper cosmological bounds 1 . These upper mass bounds, however, rely on the assumption that the neutrino sector hides no additional surprises. Specifically, that a neutrino per photon ratio has been fixed since the decoupling of the active neutrinos and the subsequent $e^{+} e^{-}$ annihilation. Or that neutrinos do not couple to unseen fields 23 . These assumptions are verifiable by cosmological observations.

Perturbations in the cosmic microwave background (CMB) carry imprints of any species which contributed to the universe density when the perturbations reentered the horizon ${ }^{\mathrm{a}}$. Some of these imprints cannot be mimicked by changes of the standard cosmological parameters. Moreover, they reveal the internal interactions of species which couple only gravitationally to the visible sector. The big bang nucleosynthesis (BBN) yield of light elements, notably $\mathrm{He}^{4}$, is as well sensitive to all the dominant species in the radiation epoch, but at high redshifts $z \sim 10^{9}-10^{8}(\sim 100 \mathrm{keV})$. The CMB peaks, on the other hand, probe the late radiation era ${ }^{\mathrm{b}} z \sim 10^{4}-10^{3}(\sim 10-1 \mathrm{eV})$.

The first WMAP data release 4 places the effective number of relic neutrinos in a 2- $\sigma$ range $1 \lesssim N_{\nu} \lesssim 7$, mildly improved ${ }^{5}$ by complementary probes. The range will be narrowed by an order of magnitude and more as the CMB sky is mapped at smaller angles: at $1 \sigma, \mathrm{tc} \frac{617}{} \Delta N_{\nu} \approx 0.2$ by PLANCK and up to $\Delta N_{\nu} \approx 0.05$ by more advanced proposed

\footnotetext{
${ }^{*}$ Based on the proceedings for the 10th Int. Symposium on Particles, Strings and Cosmology (PASCOS 04) at Northeastern University, Boston, August 16-22, 2004.

aWe say that a perturbation mode "reentered the horizon" when the Hubble scale $H^{-1}$ exceeded the mode wavelength.

${ }^{\mathrm{b}}$ The redshifts probed by the CMB multipoles with $l \leq 1500[\lesssim 3000]$ are $z_{l \text {,entry }} \lesssim 3 \times 10^{4}\left[\lesssim 6 \times 10^{4}\right]$, assuming the concordance $\Lambda$ CDM model.
} 
missions.

\section{Imprints of neutrino gravity on CMB}

The CMB fluctuations could not escape the gravity of relic neutrinos during the radiation era, when neutrinos were among the dominant species. The neutrino fraction $R_{\nu} \equiv \rho_{\nu} / \rho_{r}$ of the total radiation energy density $\rho_{r}=\rho_{\gamma}+\rho_{\nu}=\rho_{\gamma} /\left(1-R_{\nu}\right)$ is $41 \%$ in the standard scenario. With the present cosmological bounds ${ }^{1} m_{\nu} \lesssim 0.14$ to $0.6 \mathrm{eV}$, the standard neutrinos were all highly relativistic in the radiation era, prior to matter-radiation density equality at

$$
z_{\mathrm{eq}}+1=\frac{\rho_{c+b, 0}}{\rho_{\gamma, 0}}\left(1-R_{\nu}\right) \simeq 3.3 \times 10^{3} \frac{\Omega_{c+b} h^{2}}{0.14} \frac{1.69}{1+0.23 N_{\nu}} .
$$

[Here, $c+b$ labels cold dark matter $(\mathrm{CDM})$ plus baryons, "0" denotes the present values, and the last two fractions are close to unity in the standard $\Lambda \mathrm{CDM}+3 \nu$ scenario.] These neutrinos become non-relativistic below the redshift $z_{m_{\nu}} \approx m_{\nu} /\left(3 k T_{\nu, 0}\right) \simeq 200 m_{\nu} /(0.1 \mathrm{eV})$, i.e., only after the CMB decoupling $\left(z_{\gamma \mathrm{d}} \approx 1090\right)$.

The growth of cosmological structure in the matter era is also affected by neutrino mass density. It carries signatures of a neutrino free streaming scale ${ }^{8}$, which by then is sensitive to $m_{\nu}$. Although the low neutrino masses influence the perturbation dynamics only after the $\mathrm{CMB}$ last scattering, they may in future be probed by CMB due to their impact on large-scale structure and induced by it CMB lensing .

The impact of relic neutrinos cannot be ignored in the search for primordial gravity waves (hence, probing the energy scale of inflation) through $B$-polarization of CMB 10 . Interaction of neutrino perturbations with tensor gravity waves suppresses the tensor amplitude on the scales entering the horizon in the radiation era $(l>200)$ and reduces the $B$-mode spectrum by as much as 11 . Even on the largest angular scales neutrinos damp the tensor power by about 111 .

In the rest of this section we review the signatures of the abundant in the radiation era neutrinos in the CMB temperature anisotropy and E-polarization.

\subsection{Background effects}

According to Friedmann equation, higher neutrino density speeds up the Hubble expansion in the radiation era. For fixed density $\Omega_{c+b} h^{2}\left(\propto \rho_{c+b, 0}\right)$, the faster expansion would reduce the size of the $\mathrm{CMB}$ acoustic horizon. In addition, a larger fraction of radiation density prior to the CMB last scattering would enhance the first acoustic peak by a stronger early integrated Sachs-Wolfe effect from the increased proximity of the radiationmatter transition (11) to the last scattering and by lesser suppression of the peak by matter perturbations $12 \mathrm{c}$.

c The accepted today interpretation of an order of magnitude larger CMB power on the scales entering the horizon before versus after the equality as the resonance driving of radiation perturbations by their self-gravity is incorrect. This can be demonstrated by the following example: Suppose that long after the superhorizon freezout of perturbations but before their horizon reentry in the radiation era, photons and neutrinos had become subdominant to an unperturbed decoupled component $X$ with the radiation equation of state $p_{X}=\rho_{X} / 3$. In this scenario, in which the metric is unperturbed and no gravitational driving of CMB 
Although the change of the acoustic horizon and the consequences of the shifted $z_{\text {eq }}$ are frequently mentioned in the literature, neither of these effects can serve as a neutrino probe. Indeed, our knowledge of other densities, in particular CDM, is likewise derived from the observed correlations of cosmological inhomogeneities. Even when $\rho_{\nu}$ is not equal to its assumed value, the angular acoustic horizon scale and $z_{\text {eq }}$ remain essentially unchanged when at any $z$ the ratio $\rho_{b} / \rho_{\gamma}$, hence the photo-baryon sound speed, has the standard value while the densities $\rho_{c+b}, \rho_{\text {dark }}$ [and $\rho_{K, 0} \equiv 3(1-\Omega) H_{0}^{2} /(8 \pi G)$ for non-flat models] are rescaled in proportion to $\rho_{r}=\rho_{\gamma}+\rho_{\nu}$.

The observed matter power $P_{m}(k / h)$ remains almost unchanged as well. ${ }^{\mathrm{e}}$ (Small changes are induced by variations in $\rho_{b} / \rho_{c}$, in, so far undetected, signatures of neutrino masses, and in the impact of neutrino perturbations, Sec. 2.2.) There is a simple explanation: In the compared scenarios most of the characteristic time and distance intervals differ by the same factor. This conformal rescaling preserves measured angles and redshifts 7 .

Several consequences of the common density rescaling can still be observed. First, a different value of the Hubble constant $H_{0}^{2} \propto \sum_{a, K} \rho_{a, 0}$. Constraints on $H_{0}$, however, tend to be weak.

Second, the scale of Silk damping ${ }^{15} \lambda_{\text {Silk }}$ is determined by the mean time of photon collisionless flight $\tau_{c}=\left(a n_{e} \sigma_{\gamma e}\right)^{-1}$, not affected by the density rescaling. (We use comoving scales and conformal times.) A larger ratio of the damping scale to the Hubble radius $\lambda_{\text {Silk }} / \mathcal{H}^{-1} \sim \sqrt{\tau_{c} \mathcal{H}}$ in models with greater $\rho_{\gamma}$ leads to increased damping of small-scale CMB anisotropies and helps to constrain $N_{\nu}$ (Table III in Ref. [7]). However, the density of free electrons $n_{e}$ around the photon decoupling, when only hydrogen was ionized, depends (for known $\rho_{b}$ ) on the primordial helium abundance $Y_{p}: n_{e} \equiv x_{e} n_{H}=x_{e}\left(1-Y_{p}\right) \rho_{b} / m_{H}$. Taking for the present uncertainty $Y_{p}=0.24 \pm 0.01^{16}, Y_{p}$ can be freely adjusted to rescale $\lambda_{\text {Silk }}$ in proportion to $\mathcal{H}^{-1}$ for $\Delta N_{\nu} \lesssim 0.4$. A detailed analysis ${ }^{7}$ ) shows that the rescaling $1-Y_{p} \propto$ $\rho_{r}^{1 / 2}$ yields almost degenerate $x_{e}(z)$, hence equivalent CMB decoupling, and unchanged $\lambda_{\text {Silk }} / \mathcal{H}^{-1}$.

\subsection{Perturbation effects}

Finally, the conformal degeneracy of CMB and matter spectra in a faster expanding background is broken by the gravitational impact of neutrino perturbations. Relative perturbation of the number density $n_{\gamma}$ of photons (coupled to baryons) evolves in the perturbed metric as

$$
\ddot{d}_{\gamma}+\frac{R_{b} \mathcal{H}}{1+R_{b}} \dot{d}_{\gamma}-\frac{1}{3\left(1+R_{b}\right)} \nabla^{2} d_{\gamma}=\nabla^{2}\left(\Phi+\frac{1}{1+R_{b}} \Psi\right) .
$$

Here $\Phi$ and $\Psi$ parameterize scalar metric perturbations $d s^{2}=a^{2}\left[-(1+2 \Phi) d \tau^{2}+(1-2 \Psi) d x^{2}\right]$,

is expected, the acoustic CMB oscillations after the horizon reentry would have precisely the same amplitude as in the model dominated by a photon fluid. To the contrary, a similar construction for the matter era 12 shows that then the freely collapsing CDM perturbations, generating non-decaying gravitational potential, cause 25-fold suppression of the CMB power $C_{l}$ in the Sachs-Wolfe 13 matter era result.

${ }^{\mathrm{d}}$ The photon density $\rho_{\gamma}$ is fixed by COBE-measured ${ }^{14} T_{\mathrm{CMB}}$.

eThe popular rule of thumb "LSS constrains $\Omega_{m} h$ " assumes a fixed neutrino fraction of radiation density and fails in our situation. Indeed, the physical quantity probed best by the large scale structure is the ratio of the comoving Hubble scale at the matter-radiation equality $\mathcal{H}_{\mathrm{eq}}^{-1}=\left(H_{\mathrm{eq}} a_{\mathrm{eq}}\right)^{-1}$ to the present Hubble scale $H_{0}^{-1}$. With eq. (1), $\mathcal{H}_{\text {eq }} / H_{0} \sim \Omega_{c+b}^{1 / 2}\left(z_{\text {eq }}+1\right)^{1 / 2} \propto \Omega_{c+b} h\left(1-R_{\nu}\right)^{1 / 2}$. It is unchanged by the discussed (conformal) rescaling. 
overdots denote the derivatives with respect to the conformal time $\tau, \mathcal{H} \equiv \dot{a} / a, R_{b}=$ $3 \rho_{b} /\left(4 \rho_{\gamma}\right)$, and

$$
d_{\gamma} \equiv \frac{\delta \rho_{\gamma}}{\rho_{\gamma}+p_{\gamma}}-3 \Psi=3\left(\frac{\delta T_{\gamma}}{T_{\gamma}}-\Psi\right)
$$

(in the Newtonian gauge) is a general-relativistic generalization ${ }^{\mathrm{f}}$ of $\delta n_{\gamma} / n_{\gamma}$.

For the radiation era, when the CMB peaks enter the horizon, eq. (2) is easily solved 7 ) in real space with the Green's function approach ${ }^{17}$ :

$$
d_{\gamma}^{\text {(rad era })}(x, \tau)=\frac{A_{\gamma}}{2} \delta_{\operatorname{Dirac}}\left(|x|-\frac{\tau}{\sqrt{3}}\right)+\frac{\Phi+\Psi}{(x / \tau)^{2}-1 / 3},
$$

where we assume adiabatic initial conditions. After Fourier transformation to $k$-space, the delta-function term describes the famous acoustic oscillations $A_{\gamma} \cos (k \tau / \sqrt{3})$ in the photon fluid with the speed of sound $c_{s}=1 / \sqrt{3}$. Whenever the metric is perturbed at the acoustic horizon, i.e. $(\Phi+\Psi)_{|x|=\tau / \sqrt{3}} \neq 0$, the small-scale singularity of the second term in eq. (15) contributes a sine component to the subhorizon oscillations in Fourier space or, equivalently, shifts the oscillation phase.

As proven in Appendix B of [7], the metric in the adiabatic Green's function (5) is perturbed beyond the acoustic horizon if only some perturbations physically propagate faster than the acoustic speed $c_{s}$. Among the standard cosmological species, only freestreaming relativistic neutrinos support a faster speed, the speed of light. (Perturbations in early quintessence do as well. They induce an even larger phase shift per equal density ${ }^{12}$.)

${ }^{\mathrm{f}} d_{\gamma}$ of eq. (3) is a unique ${ }^{12}$ perturbation variable which a) Reduces to $\delta n_{\gamma} / n_{\gamma}$ on subhorizon scales b) Freezes beyond the Hubble horizon and c) Experiences no gravitational driving on any scale in a spatially uniform metric. (The unperturbed Robertson-Walker metric can be imposed consistently by artificially populating the universe with decoupled species $X$ which density is negligible during the generation and superhorizon freezout of the studied perturbations, and requiring that $\mathrm{X}$ subsequently become homogeneous in space and dominate the total energy density. See footnote $c$ and Ref. [12].)

The corresponding gauge-independent expression for arbitrary species $a$ reads:

$$
d_{a} \equiv 3 \zeta_{a} \equiv \frac{\delta \rho_{a}}{\rho_{a}+p_{a}}+3 D+\nabla^{2} \epsilon
$$

where $D$ and $\epsilon$ parameterize scalar perturbations of the spatial metric $\delta g_{i j}=2 a^{2}\left[D \delta_{i j}-\left(\nabla_{i} \nabla_{j}-\frac{1}{3} \delta_{i j} \nabla^{2}\right) \epsilon\right]$. Various interpretations of this variable are: in the spatially flat gauge $\left(\delta g_{i j} \equiv 0\right)$ it gives $\delta n_{a} / n_{a} \equiv \delta \rho_{a} /\left(\rho_{a}+\right.$

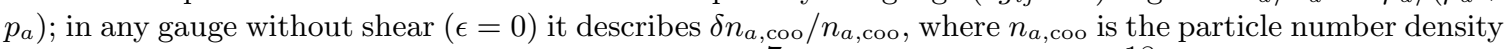
with respect to coordinate rather than proper volume ${ }^{7}$; an equivalent variable ${ }^{18} \zeta_{a}=d_{a} / 3$, a curvature perturbation on the hypersurfaces of constant $\rho_{a}$, is in widespread use to describe superhorizon dynamics of several fluids.

Eq. (2] for CMB perturbations and similar equations for other cosmological species $7 / 12$, have a series of advantages over the traditional approaches to the CMB whenever horizon-scale gravity plays a role: 1 ). The highest time derivatives of the dynamical perturbation variables now do not enter the equations implicitly through time derivatives of the "driving" potentials on the right hand side. This allows straightforward numerical integration of the equations in the convenient and fixed Newtonian gauge. 2). A direct connection 12 , to phase-space distributions in the perturbed metric. (See Refs. [8,19] for the subtleties with the traditional variables.) 3). As stated above, a change of the perturbation measure $d_{a}\left(\zeta_{a}\right)$ on any scale is caused by a physical causal mechanism only. This is not so for $\delta T_{\gamma} / T_{\gamma}, \delta \rho_{a} / \rho_{a}$, and most of the other conventional variables, unless one selects a gauge where they coincide or proportional to $d_{a}\left(\zeta_{a}\right)$. 4). Analytical solutions become simple $e^{7 \sqrt{3}}$ in this approach ${ }^{20}$ This facilitates analytical study of the physical content of CMB features and leads to new results, including those described in this review. 
This phase shift results in a non-degenerate additive shift of all the acoustic peaks in $C_{l}$, for either temperature or polarization spectra. For $N_{\nu} \approx 3$, the positions of all the peaks change as $\partial l_{\text {peak }} / \partial N_{\nu} \approx-4$.

In addition, neutrino perturbations somewhat suppress the magnitude of the acoustic oscillations while enhance the matter power 217 . With unknown primordial power, detection of this effect requires precise measurement of the matter spectrum and is difficult. Quintessence 22 perturbations cause an opposite change of the CMB to matter power ratio 2312 . If CMB finds a non-standard $N_{\nu}$, this may, in principle, discriminate between contributions of sterile particles and early quintessence.

\section{Probing particle physics}

An apparent contribution to $N_{\nu}$ may be provided by sterile neutrinos (light neutral fields which mix with the active neutrinos). Motivations to consider such fields and their status in the light of the recent oscillation data and other constraints were reviewed in detail in Cirelli's contribution 24 .

The radiation density might also be enhanced by yet unknown light particles which neither couple nor mix to the visible sector and fell off the thermal bath early, say, above the SUSY breaking scale. At that high temperature the radiation entropy was shared by a large number of relativistic species and the decoupled particles would receive only a small fraction of the (conserved) total entropy. Then the late-time contribution of the decoupled particles to the radiation density relative to the contribution of photons and active neutrinos may be small and pass the current BBN constraints.

Since the phase shift of the CMB peaks is absent when neutrinos do not free-stream, CMB will probe a growing class of models where neutrinos couple or recouple to other fields. The scenarios where recoupling takes place after BBN are not constrained 2513 by the BBN limits on $N_{\nu}$. For example, recoupling is expected if the small neutrino masses are generated by a coupling $l \nu_{R} h \phi / M$ ( $l$ and $h$ are the standard lepton and Higgs doublets and $\phi$ is a new field) after low-energy spontaneous symmetry breaking $|\phi|+\delta \phi \underline{25}$. Other neutrino mass models with neutrino coupling or recoupling have been considered 2326 . If coupled to a light field $\delta \phi$, neutrinos generally annihilate 23 after they become non-relativistic. Then neutrino mass is not bounded by the standard cosmological limits 1 . A changed phase of the acoustic peaks is a clean signatures of neutrino coupling or recoupling 25 .

\section{Summary}

Today's upper cosmological bounds on neutrino masses 1 imply that neutrinos of all the three standard generations were relativistic in the radiation era. Their energy density was comparable to that of photons, and the neutrino gravity had major impact on the cosmological dynamics. However, the induced changes of such important cosmological characteristics as the size of the acoustic CMB horizon, the redshift of radiation-matter equality, and the Hubble radius at the onset of the structure growth have no observable consequences. The corresponding angular scales and redshifts are unchanged in the scenar-

\footnotetext{
${ }^{\mathrm{g}}$ Neutrino coupling to a light field $\phi$ cannot be falsified, as claimed by the authors of Ref. [2], by the lesser large-scale structure growth due to the radiation-matter equality delay (caused by $\phi$ contribution to $\rho_{r}$ ). At least, not until we find a non-cosmological probe of CDM density. See Sec. 2.1.
} 
ios where the densities of the visible matter plus the dark matter and of the dark energy are larger in proportion to the radiation density enhancement.

This conformal degeneracy is lifted by Silk damping if the primordial helium abundance $Y_{p}$ is fixed by independent spectrophotometric measurements. However, with the uncertainty $\Delta Y_{p} \sim 0.01$, an allowed adjustment of $Y_{p}$ results in degenerate Silk damping and degenerate $\mathrm{CMB}$ decoupling among the compared scenarios for $\Delta N_{\nu}<0.4$.

The gravitational impact of neutrino perturbations on the CMB temperature and polarization angular spectra is not degenerate with any of the standard parameters. Free streaming of neutrino perturbations faster than the acoustic speed causes a measurable shift of the acoustic oscillation phase. The multipoles of all the acoustic peaks shift by an approximately same interval $\Delta l \approx-4 \Delta N_{\nu}$ (at $N_{\nu} \approx 3$ ). This shift does not occur or is reduced in the models where all or some of the neutrino types either had not decoupled or recoupled when the perturbations entered the horizon. (Similar changes might be induced by isocurvature modes, which detection would be just as exciting.)

A canonical dynamical field (quintessence), perturbations of which propagate at the speed of light, causes a similar uniform shift ${ }^{\mathrm{h}}$ of the peaks. Early quintessence may be distinguished from extra light relativistic particles by accurate measurement of the CMB to matter power ratio. Perturbations of a classical field and decoupled particles change this ratio in opposite directions.

Ultimately, neither dark matter nor dark energy entered the cosmological scene with strong theoretical motivation. The upcoming CMB data will testify whether or not the radiation epoch conceals its own surprises.

\section{Acknowledgments}

I thank the organizers of PASCOS 04, and I am grateful to S. Shankaranarayanan and A. Smirnov for their suggestions during the preparation of this contribution.

\section{References}

1) D. N. Spergel et al., Astrophys. J. Suppl. 148, 175 (2003);

S. Hannestad, JCAP 0305, 004 (2003);

S. W. Allen et al. (report $m_{\nu}=0.19_{-0.09}^{+0.10} \mathrm{eV}$ ) MNRAS 346, 593 (2003);

M. Tegmark et al., Phys. Rev. D 69, 103501 (2004);

V. Barger, D. Marfatia and A. Tregre, Phys. Lett. B 595, 55 (2004);

P. Crotty, J. Lesgourgues and S. Pastor, Phys. Rev. D 69, 123007 (2004);

U. Seljak et al., astro-ph/0407372,

G. L. Fogli et al.. hep-ph/0408045.

For a recent review see S. Hannestad, hep-ph/0409108.

2) J. F. Beacom, N. F. Bell and S. Dodelson, Phys. Rev. Lett. 93, 121302 (2004).

3) Z. Chacko, L. J. Hall, S. J. Oliver and M. Perelstein, hep-ph/0405067.

4) C. L. Bennett et al., Astrophys. J. Suppl. 148, 1 (2003).

\footnotetext{
${ }^{\mathrm{h}}$ For the quintessence energy density which in the radiation era equals the density of $N_{Q}$ effective fermions, $\Delta l \approx-11 \Delta N_{Q} 12$.
} 
5) P. Crotty, J. Lesgourgues and S. Pastor, Phys. Rev. D 67, 123005 (2003);

E. Pierpaoli, MNRAS 342, L63 (2003);

V. Barger et al., Phys. Lett. B 566, 8 (2003);

S. Hannestad, JCAP 0305, 4 (2003).

6) R. E. Lopez et al., Phys. Rev. Lett. 82, 3952 (1999);

R. Bowen et al., Mon. Not. Roy. Astron. Soc. 334, 760 (2002).

7) S. Bashinsky and U. Seljak, Phys. Rev. D 69, 083002 (2004).

8) J. R. Bond and A. S. Szalay, Astrophys. J. 274, 443 (1983).

9) M. Kaplinghat, L. Knox and Y. S. Song, Phys. Rev. Lett. 91, 241301 (2003).

10) M. Kamionkowski, A. Kosowsky and A. Stebbins, Phys. Rev. Lett. 78, 2058 (1997);

U. Seljak and M. Zaldarriaga, Phys. Rev. Lett. 78, 2054 (1997).

11) S. Weinberg, Phys. Rev. D 69, 023503 (2004).

12) S. Bashinsky, astro-ph/0405157.

13) R. K. Sachs and A. M. Wolfe, Astrophys. J. 147, 73 (1967).

14) J. C. Mather et al., Astrophys. J. 420, 439 (1994).

15) J. Silk, Astrophys. J. 151, 459 (1968).

16) K. A. Olive and G. Steigman, Astrophys. J. Suppl. 97, 49 (1995);

K. A. Olive, E. Skillman and G. Steigman, Astrophys. J. 483, 788 (1997);

Y. I. Izotov, T. X. Thuan and V. A. Lipovetsky, Astrophys. J. Suppl. 108, 1 (1997);

Y. I. Izotov and T. X. Thuan, Astrophys. J. 500, 188 (1998);

Y. I. Izotov et al., Astrophys. J. 527, 757 (1999);

M. Peimbert, A. Peimbert and M. T. Ruiz, Astrophys. J. 541, 688 (2000);

A. Peimbert, M. Peimbert and V. Luridiana, Astrophys. J. 565, 668 (2002);

Y. I. Izotov and T. X. Thuan, Astrophys. J. 602, 200 (2004);

K. A. Olive and E. D. Skillman, astro-ph/0405588

17) S. Bashinsky and E. Bertschinger, Phys. Rev. Lett. 87, 081301 (2001);

Phys. Rev. D 65, 123008 (2002).

18) D. Wands et al., Phys. Rev. D 62, 043527 (2000).

19) C. P. Ma and E. Bertschinger, Astrophys. J. 455, 7 (1995).

20) Eq. (2) may also be used off-label for quick derivation of traditional formulas. See

S. Dodelson, Modern cosmology, Academic Press (2003), Eqs. (8.20)-(8.26).

21) W. Hu and N. Sugiyama, Astrophys. J. 471, 542 (1996).

22) C. Wetterich, Nucl. Phys. B 302, 668 (1988);

B. Ratra and P. J. E. Peebles, Phys. Rev. D 37, 3406 (1988);

P. G. Ferreira and M. Joyce, Phys. Rev. Lett. 79, 4740 (1997);

I. Zlatev, L. M. Wang and P. J. Steinhardt, Phys. Rev. Lett. 82, 896 (1999).

23) P. G. Ferreira and M. Joyce, Phys. Rev. D 58, 023503 (1998);

M. Doran, M. J. Lilley, J. Schwindt and C. Wetterich, Astrophys. J. 559, 501 (2001);

R. R. Caldwell et al., Astrophys. J. 591, L75 (2003).

24) M. Cirelli, astro-ph/0410122,

M. Cirelli, G. Marandella, A. Strumia and F. Vissani, hep-ph/0403158

25) Z. Chacko, L. J. Hall, T. Okui and S. J. Oliver, hep-ph/0312267

26) N. Arkani-Hamed and Y. Grossman, Phys. Lett. B 459, 179 (1999);

T. Okui, hep-ph/0405083. 